\title{
Association between macronutrient intakes during pregnancy and risk of giving birth to small for gestational age (SGA) infants
}

\author{
S.S. Sharma ${ }^{1}$, D.C. Greenwood ${ }^{2}$, N.A.B. Simpson ${ }^{3}$ and J.E. Cade ${ }^{1}$ \\ ${ }^{1}$ Nutritional Epidemiology Group, School of Food Science and Nutrition, University of Leeds, Leeds LS2 9JT, UK, \\ ${ }^{2}$ Biostatistics Unit, Centre for Epidemiology and Biostatistics, University of Leeds, Worsley Building, Leeds LS2 9JT, \\ UK and ${ }^{3}$ Division of Obstetrics and Gynaecology, Leeds General Infirmary, Leeds LS9 9NS, UK
}

Macronutrient intake in pregnancy may contribute to adverse birth outcomes ${ }^{(1,2)}$. The aim of our analysis was to explore the association between maternal macronutrient intakes and their individual components in the first trimester, with the risk of giving birth to SGA infants.

A prospective cohort in Leeds ${ }^{(3)}$, UK of 1276 pregnant women aged $18-45$ years provided a 24 h dietary recall. Smoking habits and alcohol consumption was assessed by questionnaire. SGA was defined as birthweight below $10^{\text {th }}$ centile on a customised centile chart which was adjusted by confounding factors affecting birth weight such as maternal weight, height, ethnicity, parity at booking, gestational age at delivery and gender of neonates. Logistic regression analysis explored whether macronutrient intakes in the first trimester and their individual components was associated with risk of SGA. Models were unadjusted (model 1) and adjusted for other energy contributing nutrients, alcohol and smoking (model 2).

We found no significant associations between a particular macronutrient and their individual components with SGA, when they were adjusted for smoking and alcohol intake.

\begin{tabular}{|c|c|c|c|c|c|c|}
\hline \multirow[b]{2}{*}{ Trimester 1 macronutrient intakes (1 g/day) } & \multicolumn{3}{|c|}{ Risk of SGA, Model 1} & \multicolumn{3}{|c|}{ Risk of SGA, Model 2} \\
\hline & Unadjusted $^{\mathrm{a}} \mathrm{OR}$ & $95 \% \mathrm{CI}$ & $p$ value & Adjusted ${ }^{\mathrm{a}, \mathrm{b}} \mathrm{OR}$ & $95 \% \mathrm{CI}$ & $\overline{p \text { value }}$ \\
\hline Total protein (g/day) & 0.99 & 0.99 to 1.00 & 0.60 & 1.00 & 0.99 to 1.00 & 0.71 \\
\hline Total carbohydrate (g/day) & 0.99 & 0.99 to 1.00 & 0.64 & 0.99 & 0.99 to 1.00 & 0.68 \\
\hline - Starch (g/day) & 1.00 & 0.99 to 1.00 & $0 \cdot 25$ & 1.00 & 0.99 to 1.00 & $0 \cdot 25$ \\
\hline - Sucrose (g/day) & 1.00 & 0.99 to 1.00 & 0.05 & 1.00 & 0.99 to 1.00 & $0 \cdot 22$ \\
\hline Total fat (g/day) & $1 \cdot 00$ & 1.00 to 1.00 & 0.02 & 1.00 & 0.99 to 1.00 & $0 \cdot 14$ \\
\hline - Saturated fat (g/day) & 1.00 & 1.00 to 1.01 & 0.09 & 1.01 & 0.99 to 1.03 & $0 \cdot 17$ \\
\hline -PUFA (g/day) & 1.01 & 0.99 to 1.02 & 0.06 & 1.02 & 0.99 to 1.05 & 0.06 \\
\hline - MUFA (g/day) & 1.00 & 0.99 to 1.01 & $0 \cdot 16$ & 0.98 & 0.95 to 1.00 & $0 \cdot 19$ \\
\hline
\end{tabular}

${ }^{\mathrm{a}}$ Adjusted using the customised growth charts for maternal weight, height, ethnicity, parity, gestational age at delivery, sex of baby ${ }^{\mathrm{b}}$ Additional adjustment for other energy contributing nutrients, and average alcohol intake and smoking status during first trimester.

This may have implications for advice given to women in pregnancy about their diet, suggesting that apart from alcohol, no one source of energy is associated with greater risk of giving birth to small for gestational age (SGA) infants.

1. Imdad A \& Bhutta ZA (2011) Public Health 11, 1-9.

2. CucÓ G, Arija V, Iranzo R et al. (2006) Acta Obstet Gynecol Scand 85, 413-21.

3. Boylan SM, Cade JE, Kirk SFL et al. (2008) Br J Nutr 100, 875-82. 